\title{
Taurine-EVA copolymer-paraffin rods dosimeters for EPR high-dose radiation dosimetry
}

Ahmed M. Maghraby,

A. Mansour,

A. A. Abdel-Fattah

\begin{abstract}
Taurine/EPR rods $(3 \times 10 \mathrm{~mm})$ have been prepared by a simple technique in the laboratory where taurine powder was mixed with a molten mixture of paraffin wax and an ethylene vinyl acetate (EVA) copolymer. The binding mixture EVA/Paraffin does not present interference or noise in the EPR signal before or after irradiation. The rods show good mechanical properties for safe and multi-use handling. An EPR investigation of radiation induced radicals in taurine rods revealed that there are two types of radicals produced after exposure to gamma radiation $\left({ }^{60} \mathrm{Co}\right)$. EPR spectra were recorded and analyzed - also the microwave power saturation and modulation amplitude were studied and optimized. Response of taurine to different radiation doses (1.5-100 kGy) was studied and found to follow a linear relationship up to $100 \mathrm{kGy}$. Radiation induced radicals in taurine persists and showed a noticeable stability over 94 days following irradiation. Uncertainities associated with the evaluation of radiation doses using taurine dosimeters were discussed and tabulated. It was found that taurine possesses good dosimetric properties using EPR spectroscopy in high doses in addition to its simple spectrum.
\end{abstract}

Key words: EPR $\bullet$ ESR $\bullet$ radiation dosimetry $\bullet$ taurine $\bullet$ alanine

A. M. Maghraby ${ }^{凶}$

Radiation Dosimetry Department,

National Institute of Standards (NIS),

12211 Tersa Str., Haram-Giza, P.O. Box 136, Egypt

and Faculty of Science and Humanities,

Physics Department,

Salman bin Abdulaziz University,

Alkharj, Saudi Arabia,

Tel.: +2 012450 9723, Fax: +2 023386 7451,

E-mail: maghrabism@yahoo.com

A. Mansour, A. A. Abdel-Fattah

National Center for Radiation Research and Technology,

Atomic Energy Authority,

Nasr City 11371, P. O. Box 8029, Cairo, Egypt

Received: 30 October 2013

Accepted: 3 February 2014

\section{Introduction}

Ionizing radiation dose assessment using an electron paramagnetic resonance (EPR) spectrometer increases widely several applications as it enables re-evaluation of radiation dosimeters. Also, it provides easy and fast procedures for dose estimation, without the need for complicated long practical procedures. Alanine/EPR is considered as the main dosimetry system for industrial applications since 1962 [1, 2]. However, alanine possesses a complex time dependence of the EPR spectrum of its radiation-induced radicals [3] in addition to its complicated spectrum which is attributed to not less than three radicals [4-6] and its limited sensitivity to low doses and hence it possesses reasonable large uncertainties [7].

Finding a new EPR dosimetry system for high radiation doses is a challenge, as the proposed material should show high stability of its radiation-induced radicals; this is beside its linear response over a wide range of radiation doses [8-10]. There are several attempts of finding new EPR dosimetry systems; none of them was able to replace alanine $[11,12]$.

Taurine was suggested as a promising radiation dosimeter using EPR spectroscopy [13] it is characterized by its simple spectrum and hence easy quantification, in addition to the marked stability of its radiation-induced radicals. As taurine is non-toxic, it could easily replace alanine in food irradiation dosimetry as well as some medical applications. 
Taurine exposure to gamma radiation results in a singlet EPR signal which is attributed to the formation of sulphur trioxide anions $\left(\mathrm{SO}_{3}^{-}\right)$. There are two distinctive magnetically active centres in irradiated taurine and both of them belong to radicals of both isotopes of sulphur (S-32 and S-33) [14].

In the current work, we are taking a step further toward establishing a new EPR dosimeter in the form of rods made of taurine mixed with an ethylene vinyl acetate copolymer (EVA), copolymer and paraffin. Prepared dosimeters are of different taurine concentrations and are investigated from the radiation dosimetry point of view using EPR.

\section{Materials, instruments and methods}

\section{Materials}

Radiation sensitive material used in the production of dosimeters rods is taurine which is also named as 2-aminoethansulphonic acid, it was purchased from $\mathrm{Ox}$ ford laboratory, India, in the form of white fine crystals. Taurine has the molecular formula $\left(\mathrm{C}_{2} \mathrm{H}_{7} \mathrm{NO}_{3} \mathrm{~S}\right)$, with a molecular mass of $125.15 \mathrm{~g} \cdot \mathrm{mol}^{-1}$, the mass density is $1.734 \mathrm{~g} \cdot \mathrm{cm}^{-3}$ and the melting point is $305.11^{\circ} \mathrm{C}$. The molecular structure diagram is represented in Fig. 1. The binding material is composed of hot melt stick adhesive based on the ethylene vinyl acetate copolymer (Tec-Bond 232/12, Power Adhesives Limited, England) and paraffin wax (congealing point $65-71^{\circ} \mathrm{C}, \mathrm{BHD}$ ).

\section{Preparation of dosimeter rods}

An equal weight of a mixture of paraffin wax and ethylene vinyl acetate (EVA) hot-melt adhesive was melted in a crucible at $358.15-368.15 \mathrm{~K}$ in a water bath. EVA shows a complete compatibility with paraffin wax 5, 10 and $20 \%$ fine powdered taurine material was added (w/w) to the hot mixture suspension and mechanically stirred for about $10 \mathrm{~min}$ at the same temperature to obtain a homogeneous mixture [15]. The hot suspension was sucked into polypropylene tubes (inner diameter $3 \mathrm{~mm}$ ) and was left to solidify by cooling the taurine mixture. Rods were obtained by removing the polypropylene tube, and then cutting into rods $(3 \times 10 \mathrm{~mm}$ dimensions). The average mass of the newly made taurine dosimeters were found to be $0.0726 \pm 0.0083 \mathrm{~g}$ for a concentration of $5 \%, 0.0798 \pm 0.0102 \mathrm{~g}$ for a concentration of $10 \%$ and $0.0869 \pm 0.0126 \mathrm{~g}$ for a concentration of $20 \%$, and hence there are three different types of rods prepared depending on taurine concentration, namely EVAPT05, EVAPT10 and EVAPT20 containing 5, 10 and $20 \%$ of taurine, respectively.

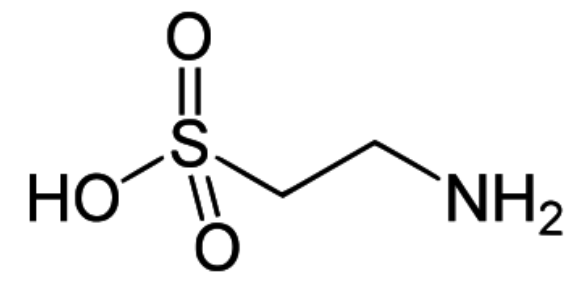

Fig. 1. Molecular structure of taurine.

\section{Irradiation processes}

The irradiation of taurine rods was carried out using $\gamma$-rays in a ${ }^{60} \mathrm{Co}$ gamma cell 220 Excel at the central spatial position which is the most uniform position isodose in the chamber, and is accurately calibrated using standard NPL alanine reference dosimeters. The temperature during $\gamma$-ray irradiation was adjusted to $308 \mathrm{~K}$. The absorbed dose rate at the time of irradiation was $3.26 \mathrm{kGy} / \mathrm{h}$. At least three rods for each dose were irradiated together at the central position of the sample chamber using a specially designed holder made of polystyrene to ensure electronic equilibrium. Radiation doses (air Kerma) delivered to samples ranged from 0.1 to $100 \mathrm{kGy}$.

\section{EPR measurements}

The EPR spectra of unirradiated and irradiated taurine rods were recorded using an EMX spectrometer (X-band, $\sim 9.5 \mathrm{GHz}$ ) the cavity used was the standard Bruker ER 4102 rectangular cavity. The operating conditions for the EPR spectrometer were as follows: microwave power $2.012 \mathrm{~mW}$, modulation amplitude $0.5 \mathrm{mT}$, time constant $81.92 \mathrm{~ms}$ and conversion time $20.48 \mathrm{~ms}$. Samples were inserted in EPR tubes and measured at the above instrumental parameters. The bottom of the EPR tube was adjusted at a fixed position in order to ensure reproducible and accurate positioning of the rods in the most sensitive volume of the cavity. The peak-to-peak height of the radiation-induced EPR first derivative signals was measured for each sample. To obtain high reproducibility in measurements each rod was marked and placed in a fixed orientation during spectra acquisition. All EPR measurements were carried out at the laboratory temperature $(298 \pm 2 \mathrm{~K})$. Also EPR spectra were recorded at two perpendicular orientations for each rod in the EPR cavity $\left(\mathrm{P}_{0}\right.$, and $\left.\mathrm{P}_{90}\right)$ and each spectrum is of a single scan $(n=1)$. The response curves were established in terms of signal height of the dosimetric peak (average peak-to-peak heights, $H_{\mathrm{PP}}$ of the two orientations $\mathrm{P}_{0}$ and $\mathrm{P}_{90}$ ) divided by sample mass as a function of irradiation dose in kGy. Stability of the EPR spectrometer sensitivity was checked before and after each series of measurements using the standard reference material (DPPH, $\alpha, \alpha$-diphenyl $\beta$-picrylhydrazyl) also empty tube spectra were acquired before recording sample spectra in order to ensure the purity of the obtained signals.

\section{Results and discussion}

\section{Spectral features}

No distinctive features were observed in EPR spectra of unirradiated dosimeter rods, however, the spectrum of $3 \mathrm{kGy}$ gamma irradiated taurine possesses two distinct signals, namely $S_{1}$ and $S_{2}$ as shown in Fig. 2, where $S_{1}$ is a singlet and lies at $g\left(S_{1}\right)=2.00973 \pm 0.00042$ and its line-width, $W_{\mathrm{PP}}\left(\mathrm{S}_{1}\right)=1.05 \mathrm{mT}$. $\mathrm{S}_{1}$ is attributed to the formation of the sulphur trioxide anion $\left(\mathrm{SO}_{3}^{-}\right)$as a result of irradiation, while $\mathrm{S}_{2}$ may be attributed to the presence of another type of radicals of different origin. 


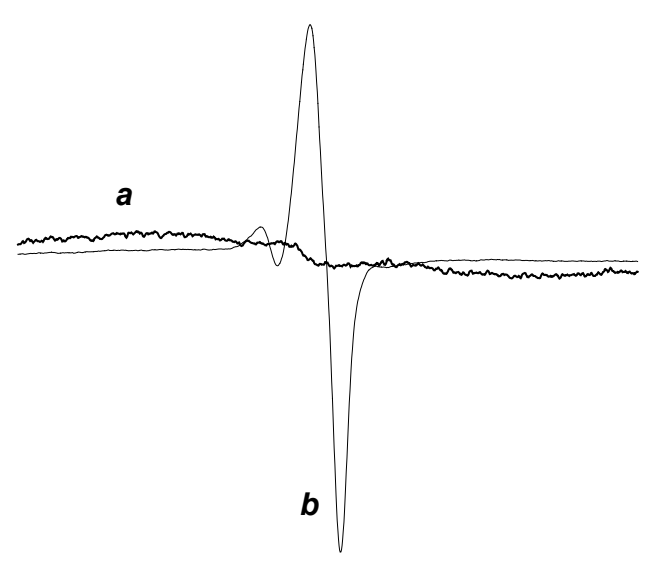

Fig. 2. (a) EPR spectrum of non-irradiated EVAPT20 rods, (b) EPR spectrum of EVAPT20 rods irradiated to $3 \mathrm{kGy}$.

Figures $3 \mathrm{a}, 3 \mathrm{~b}$ and $3 \mathrm{c}$ shows that the peak-to-peak height of $\mathrm{S}_{1}$ increases as the radiation absorbed dose increases, while $\mathrm{S}_{2}$ does not exhibit any increase of peak-to-peak height, and this confirms that both $S_{1}$ and $S_{2}$ are of different origin. Figure $3 \mathrm{c}$ represents the same EPR spectrum in Figs. 3a and $3 \mathrm{~b}$ but acquired at much higher dose $(50 \mathrm{kGy})$, where $S_{1}$ becomes much stronger than $S_{2}$ and this leads $S_{2}$ to become less noticeable, and hence the dosimeter spectrum appears as a single peak only. Based
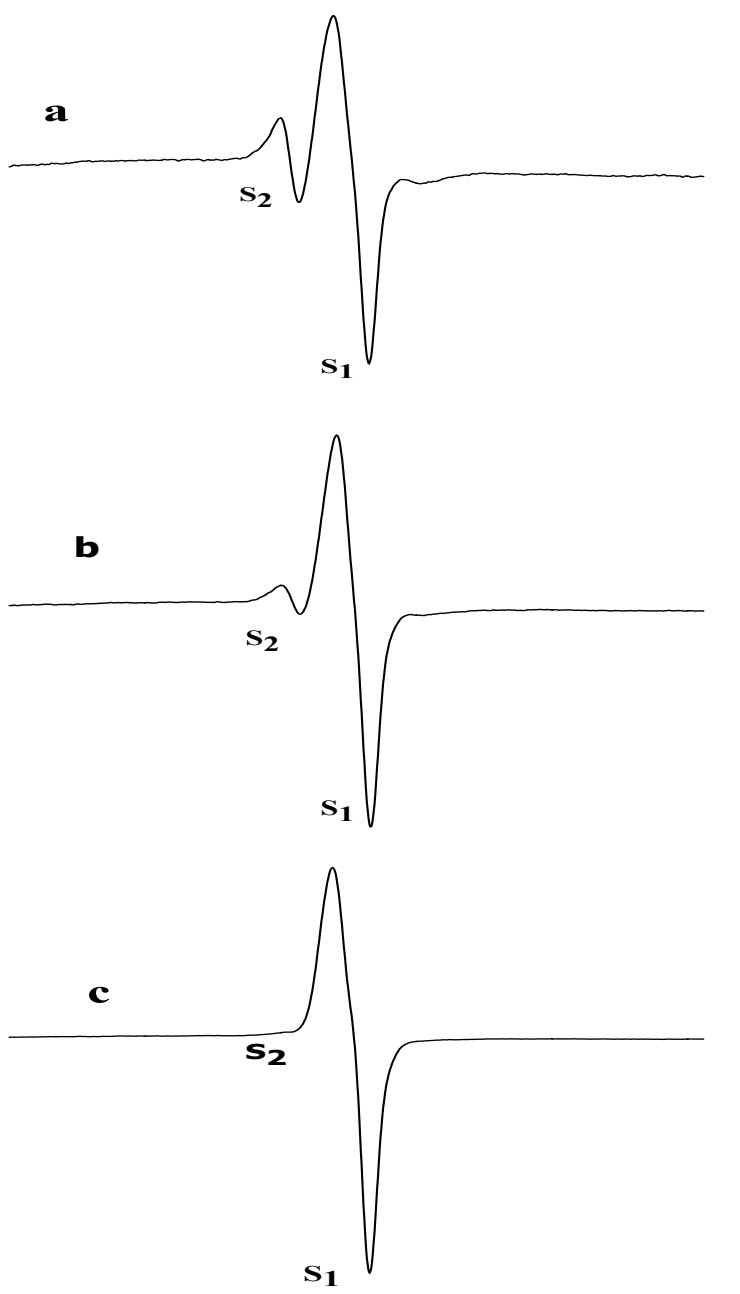

Fig. 3. EPR spectra of irradiated taurine samples (EVAPT20). EPR spectrum at $1.5 \mathrm{kGy}(\mathrm{a}), 3 \mathrm{kGy}(\mathrm{b})$, and $50 \mathrm{kGy}$ (c). on these preliminary features of $S_{1}$, it was considered as a dosimetric signal and hence current work focus is restricted to $S_{1}$ as $S_{2}$ is of minor importance from the dosimetric point of view.

\section{Response to the change in microwave power and modulation amplitude}

The relation between peak-to-peak height $\left(H_{\mathrm{PP}}\right)$ and the microwave power square root for signal $S_{1}$ is similar to that in the case of pure taurine studied previously [13] where $H_{\mathrm{PP}}\left(\mathrm{S}_{1}\right)$ responds to the change in microwave power in a linear regression until the value of $2.012 \mathrm{~mW}$ (corresponding to $P^{1 / 2}=1.4184 \mathrm{~mW}^{1 / 2}$ ). In order to avoid power saturation, the value of $2.012 \mathrm{~mW}$ has been selected for carrying out measurements which are almost at the upper end of the linear range of the dependence.

The effect of modulation amplitude on the peak-to-peak height $\left(H_{\mathrm{PP}}\right)$ of $\mathrm{S}_{1}$ is similar to taurin powder case where $H_{\mathrm{PP}}\left(\mathrm{S}_{1}\right)$ increases linearly as the modulation amplitude increases up to $0.5 \mathrm{mT}$, after this value $H_{\mathrm{PP}}\left(\mathrm{S}_{1}\right)$ increases non-linearly until $1.0 \mathrm{mT}$, after which, $H_{\mathrm{PP}}\left(\mathrm{S}_{1}\right)$ tends to stabilize.

\section{Response to radiation}

The prepared taurine rods have good mechanical properties adequate for easy and safe handling. Figure 4 shows the calibration curves obtained for the irradiated EVAPT05, EVAPT10 and EVAPTA20 rods in terms of average peak-to-peak amplitude normalized to rod mass vs. the radiation doses (air kerma) over the range from 1.5 to $100 \mathrm{kGy}$. A linear relationship is clearly demonstrated, EVAPT20 response was fitted linearly according to the relation $\mathrm{Y}_{1}=0.396^{*} \mathrm{X}_{2}+0.253$, and for EVAPT10 was $Y_{2}=0.175^{*} X_{2}+0.501$, and for EVAPT05 the fitting equation was $Y_{3}=0.086 * X_{3}+0.330$. Coefficients of determination $\left(\mathrm{R}^{2}\right)$ of these fittings were $0.999,0.995$, and 0.994 , respectively.

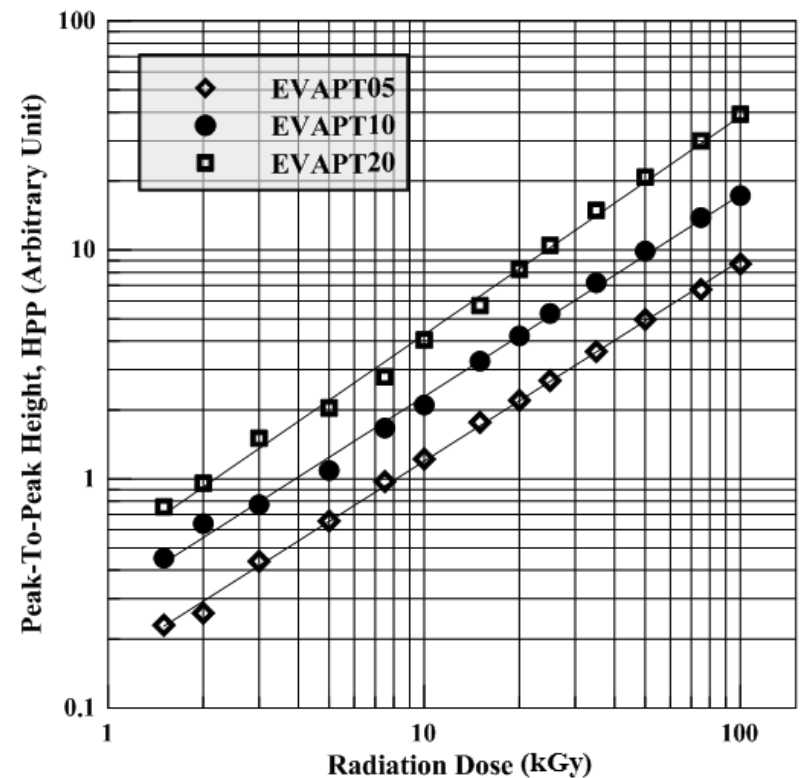

Fig. 4. Dose response curves of the EVAPT05, EVAPT10, and EVAPT20 rods at different doses from 1.5 to $100 \mathrm{kGy}$. 


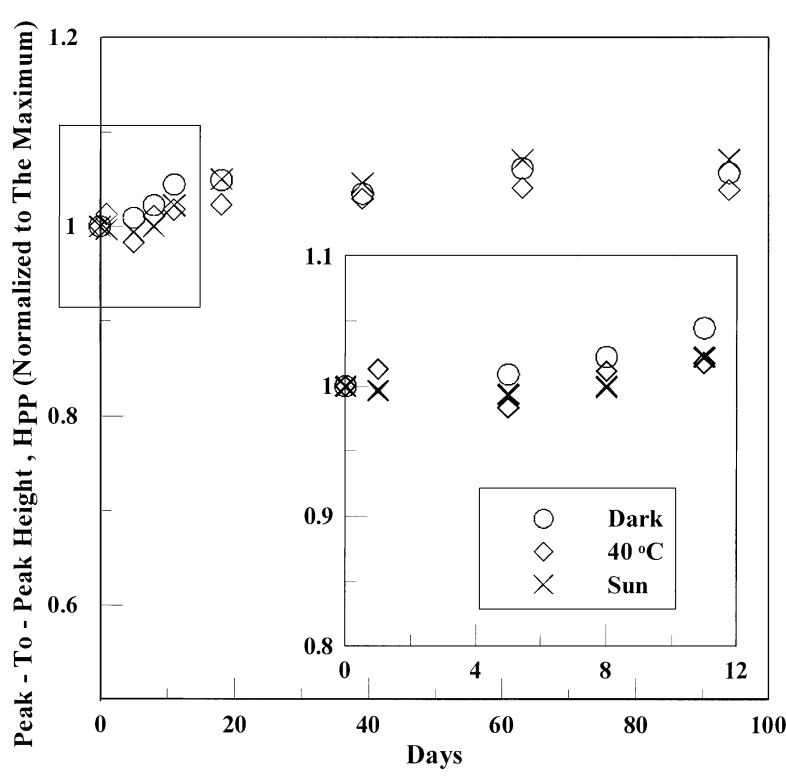

Fig. 5. Peak-to-peak heights $\left(H_{\mathrm{PP}}\right)$ of $\mathrm{S}_{1}$ as a function of post-irradiation time (days) in different conditions over 94 days after irradiation, the inset clarifies the data of the first 11 days.

\section{Stability of free radicals}

\section{Response to different doses}

One of the basic features of an EPR dosimeter is the stability of its radicals resulting from exposure to radiation; and hence it is of great importance to investigate the time dependence of those radiation-induced free radicals. Six taurine rods (EVAPT20) irradiated to six different doses: 2, 5, 10 and $25 \mathrm{kGy}$ were used to check stabilities of $S_{1}$, their EPR spectra were recorded over 94 days following irradiation. Similar to the case of taurine powder dosimeters [13] it is found that $H_{\mathrm{PP}}\left(\mathrm{S}_{1}\right)$ shows a fair stability over this period. For $2 \mathrm{kGy}$ gamma irradiated taurine dosimeters, peak-to-peak height $\left(H_{\mathrm{PP}}\right)$ was stable within $1.7 \%$ of the average signal intensity over the 94 days, while $H_{\mathrm{PP}}$ for the $5 \mathrm{kGy}$ irradiated dosimeters was stable within $2.2 \%$, and $2.3 \%$ for the
$10 \mathrm{kGy}$ irradiated dosimeters, and for the $25 \mathrm{kGy}$ gamma irradiated dosimeters $H_{\mathrm{PP}}$ showed stability within $1.5 \%$ of its average value.

\section{Impact of storage conditions}

The post-irradiation stability has been studied for 3 sets of EVAPT20 (each set consists of 3 rods) for a period of 94 days. 3 sets were irradiated to a dose of $25 \mathrm{kGy}$ and stored under different conditions: in the dark and at room temperature (about $25^{\circ} \mathrm{C}$ ), in the sunlight, and $40^{\circ} \mathrm{C}$ in an oven. Figure 5 shows the relative peak-to-peak signal height as a function of post-irradiation storage time over 94 days. Circles represent readings of dosimeters kept in the dark and at room temperature, dosimeters were stable over the 94 days period within $2 \%$, most of fluctuations in signal intensity were within the first 8 days following irradiation where signal intensity was stable within $1.9 \%$, while during the remaining 86 days fluctuations in signal intensities did not exceed $1 \%$.

On the other hand, readings of dosimeters kept in the sunlight over the same period are represented by crosses, and from Fig. 5 it is clear that signal intensity increases as time passes and this may be attribute to the emergence of new radicals after exposure of dosimeters to the sunlight and hence irradiation continues by UV rays $[16,17]$. Signal intensities increased by a factor of $1.7 \%$ at the end of the period of study. Third group of dosimeters which was kept at a temperature of $40^{\circ} \mathrm{C}$, possessed a stability of signal intensities within $3 \%$ of the avrage value over 94 days.

The minor differences between results of the three groups of dosimeters reflect the noticeable stability of the radiation-induced radicals where more detailed studies of decay kinetics may be required seperately and in details.

\section{Uncertainity}

Uncertainities associated with the evaluated radiation doses using taurine-EVA-copolymer-paraffine dosim-

Table 1. An example of the obtained uncertainity budget, this example applies for the radiation dose of $20 \mathrm{kGy}$

\begin{tabular}{|c|c|c|c|c|c|c|}
\hline Uncertainity component & Value & Distribution & Type & Divisor & $C_{i}^{\mathrm{b}}$ & $\begin{array}{l}\text { Standard } \\
\text { uncertainity }\end{array}$ \\
\hline \multicolumn{7}{|l|}{ Irradiation } \\
\hline radiation dose determination & 1.1 & Gaussian & A & 2 & 1.0 & 0.55 \\
\hline irradiation time & 0.1 & Rectangular & $\mathrm{B}$ & $\sqrt{3}$ & 0.005 & 0.0003 \\
\hline irradiation temperature & 0.2 & Gaussian & B & 1 & 0.01 & 0.002 \\
\hline \multicolumn{7}{|l|}{ Measurement } \\
\hline repeatability & 0.5 & Gaussian & A & 1 & 1.0 & 0.5 \\
\hline $\begin{array}{l}\text { vertical positioning of the dosimeters inside } \\
\text { the EPR cavity }\end{array}$ & 0.8 & Gaussian & $\mathrm{B}$ & 1 & 0.95 & 0.76 \\
\hline $\begin{array}{l}\text { angular positioning of the dosimeters inside } \\
\text { the EPR cavity }\end{array}$ & 3.0 & Triangular & $\mathrm{B}$ & $\sqrt{6}$ & 0.01 & 0.012 \\
\hline \multicolumn{7}{|l|}{ Other influencing parameters } \\
\hline balance precision & 0.2 & Rectangular & $\mathrm{B}$ & $\sqrt{3}$ & 1.0 & 0.12 \\
\hline linearity & $<0.1^{\mathrm{a}}$ & Rectangular & A & $\sqrt{3}$ & 1.0 & 0.06 \\
\hline combined uncertainity & 1.07 & Gaussian & & & & \\
\hline coverage factor, $k$ & 2 & & & & & \\
\hline expanded uncertainity & 2.15 & Gaussian & & & & \\
\hline
\end{tabular}

${ }^{\mathrm{a}}$ The value 0.1 was used for calculations. $\quad{ }^{\mathrm{b}} C_{i}-$ sensitivity coeffecient. 
etres include uncertainities in radiation doses evaluation using the refrence dosimetry system, irradiation time, and irradiation temperature. Also, the uncertainity budget includes uncertainities due to repeatability, sample positioning inside the cavity both vertically and angularly, linearity, and balance precision.

Uncertainity budget was estimated according to standard international guides [18-21]. Table 1 represents the uncertainity budget associated with radiation dose evaluation of $20 \mathrm{kGy}$ irradiated dosimeters, from the table, the combined uncertainity is equal to $1.07 \%$, while the expanded uncertainity $(k=2 ; 95 \%)=2.15 \%$.

\section{Conclusion}

Taurine EVA-copolymer paraffin rods can be prepared for the use as EPR dosimeters of different concentrations of taurine. The dosimetric signal in the EPR spectrum of these dosimeters possesses a linear response to gamma radiation over the range of doses (1.5-100 kGy). Radiation-induced radicals in taurine dosimeters show a noticeable stability over more than 90 days after irradiation, which is a sufficient time to be used as transfer dosimeter. New taurine dosimeters show good dosimetric features and are easy to be used for quantification due to their simple spectra. Further studies may be required for investigating detailed response of taurine rods dosimeters in other ranges of radiation doses and different radiation qualities.

\section{References}

1. Bradshaw, W. W., Cadena, D. G., Crawford, G. W.. \& Spetzler, H. A. W. (1962). The use of alanine as a solid dosimeter. Radiat. Res., 17, 11-21.

2. Guidelli, E. J., Ramos, A. P., Zaniquelli, M. E. D., Nicolucci, P., \& Baffa, O. (2012). Synthesis of silver nanoparticles using DL-alanine for ESR dosimetry applications. Radiat. Phys. Chem., 81(3), 301-307.

3. Nagy,V., \& Desrosiers, M. (1996) Complex time dependence of the EPR signal of irradiated L- $\alpha$-alanine. Appl. Radiat. Isot., 47, 789-793.

4. Heydari, M., Malinen, E., Hole, E., \& Sagstuen, E. (2002). Alanine radicals. 2. The composite polycrystalline alanine EPR spectrum studied by ENDOR, thermal annealing and spectrum simulations. J. Phys. Chem. A, 106, 8971-8977.

5. Malinen, E., Heydari, M., Sagstuen, E., \& Hole, E. (2003). Alanine radicals. Part 3. Properties of components contributing to the EPR spectrum of X-irradiated alanine dosimeters. Radiat. Res., 159, 23-32.
6. Sagstuen, E., Hole, E., Haugedal, S., \& Nelson, W. (1997). Alanine radicals: structure determination by EPR and ENDOR of single crystals X-irradiated at 295. J. Phys. Chem. A, 101, 9763-9772.

7. Maghraby, A. (2011). Uncertainty attributed to signal averaging in a single averaged alanine EPR spectrum for low-dose applications. Radiat. Prot. Dosim., 143(1), 6-12.

8. Maghraby, A., \& Tarek, E. (2005) A new EPR dosimeter based on sulfanilic acid. Radiat. Meas., 41, 170-176.

9. Maghraby, A. (2007). A sensetive EPR dosimetry system based on sulfamic acid. Nucl. Instrum. Methods Phys. Res. Sect. B-Beam Interact. Mater. Atoms, 262, 46-50.

10. Maghraby, A., Salama, E., \& Mansour, A. (2011). EPR/ homotaurine: A possible dosimetry system for high doses. Nucl.Instrum. Methods Phys. Res. Sect.A-Accel. Spectrom. Dect. Assoc., 659, 504-507.

11. Olsson, S., Bagherian, S., Lund, E., Carlsson, G., \& Lund, A. (1999). Ammonium tartrate as an ESR dosimeter material. Appl. Radiat. Isot., 50, 955-965.

12. Vestad, T., Malinen, E., Lund, A., Hole, E., \& Sagstuen, E. (2003). EPR dosimetric properties of formats. Appl. Radiat. Isot., 59, 181-188.

13. Maghraby, A., Mansour, A., \& Tarek, E. (2012). Taurine for EPR dosimetry. Radiat. Environ. Biophys., 51, 255-261.

14. Maghraby, A. (2012). Ionizing radiation induced radicals. In M. Nenoi (Ed.), Current topics in ionizing radiation research. InTech Publishers. Retrieved from http://www. intechopen.com/books/current-topics-in-ionizing-radiation-research/ionizing-radiation-induced-radicals. DOI: $10.5772 / 34925$

15. Soliman, Y., \& Abdel-Fattah, A. (2012). Magnesium lactate mixed with EVA polymer/paraffin as an EPR dosimeter for radiation processing application. Radiat. Phys. Chem., 81(2), 1910-1916.

16. Ciesielski, B., Schutka, K., Penkowski, M., \& Sagstuen, E. (2004). EPR study of light illumination effects on radicals in gamma-irradiated L-alanine. Spectrochim. Acta A, 60(6), 1327-1333.

17. El-Faramawy, N. (2005). Comparison of $\gamma$ - and UV-light-induced EPR spectra of enamel from deciduous molar teeth. Appl. Radiat. Isot., 62(2), 191-195.

18. Bureau International des Poids et Mesures. (2008). Evaluation of measurement data - Guide to the expression of uncertainty in measurement. JCGM 100:2008(F). Severes Cedex, France.

19. International Organization for Standardization. (1995). Guide to the expression of uncertainty in measurement. GUM:1995. Geneva.

20. Taylor, B. N, \& Kuyatt, C. E. (1994). Guidelines for evaluating and expressing the uncertainty of NIST measurement results. National Institute of Standards and Technology: Gaithersburg, MD. (NIST Technical Note 1297).

21. United Kingdom Accreditation Service. (2012). The expression of uncertainty and confidence in measurement. UKAS Publication M3003. Retrieved from http://www.caa.co.uk. 